\title{
Cortical and subcortical JC virus infection: two unusual cases of AIDS associated progressive multifocal leukoencephalopathy
}

\author{
B J Sweeney, H Manji, R F Miller, M J G Harrison, F Gray, F Scaravilli
}

\begin{abstract}
Two patients with AIDS and progressive neurological syndromes had necropsies that identified JC virus infection of the cerebral or cerebellar cortex. The unusual presentation of progressive multifocal leukoencephalopathy with grey matter involvement and normal cerebral imaging is discussed.
\end{abstract}

(F Neurol Neurosurg Psychiatry 1994;57:994-997)

Progressive multifocal leukoencephalopathy, originally described by Åström, Mancall, and Richardson in 1958, is an opportunistic infection of the CNS by the papovavirus JC. ${ }^{12}$ It begins insidiously and is accompanied by focal neurological signs that include sensory and motor abnormalities, impaired vision, dysphasia, and intellectual impairment. ${ }^{2}$ The disease progresses rapidly and death usually occurs within one year of onset.

Morphological changes include areas of white-grey or yellowish discolouration localised to the white matter, which often become confluent. ${ }^{3}$ Within these areas myelin loss, the presence of foamy macrophages, reactive or pleomorphic astrocytes and enlarged oligodendrocytes containing amphophilic intranuclear inclusions are the salient features. The use of in situ hybridisation techniques helps to confirm that the inclusion bodies are those of papovavirus. The myelin loss results from viral infection and subsequent death of oligodendrocytes. The characteristic imaging correlates of these pathological changes are asymmetric lesions of white matter that do not exert a mass effect or enhance with contrast. ${ }^{2}$ The issue of grey matter involvement in progressive multifocal leukoencephalopathy is discussed later.

Before the AIDS epidemic most cases occurred in patients who had immunodeficiency related to neoplastic, autoimmune or iatrogenic disorders. ${ }^{2}$ The incidence has increased considerably since the advent of AIDS in the early 1980s - for example, there was a fourfold increase in the incidence of progressive multifocal leukoencephalopathy as a cause of death in the United States between 1979 and $1986 .{ }^{4}$ Progressive multifocal leukoencephalopathy was first reported in a patient with AIDS by Miller et al in 1982 and is an "AIDS-defining" illness. ${ }^{5}$ Clinical series have reported the incidence of progressive multifocal leukoencephalopathy as up to $3 \%$ in patients with AIDS and as the AIDS defining illness in up to $1 \% .^{6}$ Postmortem series have given the incidence as between $2-8 \%$ in patients dying of AIDS. ${ }^{3}$ Hitherto there have been no major pathological differences reported between progressive multifocal leukoencephalopathy in AIDS and progressive multifocal leukoencephalopathy associated with other immunodeficiency diseases. ${ }^{7}$ Because of the apparently increased incidence of progressive multifocal leukoencephalopathy in AIDS compared with other immunodeficiency states it has been suggested that JC virus infection may be synergistic with HIV infection. ${ }^{8}$ For example, it has been shown that the tyrosine aminotransferase protein of HIV transactivates the JC virus in vitro. ${ }^{9}$

Whereas grey matter change associated with JC virus infection and progressive multifocal leukoencephalopathy have been reported in various clinical and pathological studies, it has been as a secondary phenomenon to underlying white matter disease. ${ }^{6710-12}$ In this paper we report two cases of AIDS associated progressive multifocal leukoencephalopathy in which JC viral infection and destruction of grey matter and adjacent subcortical white matter was the major pathological change. Both patients had progressive neurological syndromes that were extensively investigated before death without a conclusive diagnosis being made.

\section{Patients and methods}

CASE REPORTS:

Case 1 was a 32 year old man who presented in July 1991 with a history of difficulty using his right hand. He was a haemophiliac who had acquired HIV-1 infection in a blood transfusion given during a postoperative haemorrhage in 1983. He had not had any AIDS associated illnesses but had recently commenced prophylactic cotrimoxazole to prevent Pneumocystis carinii pneumonia. On clinical examination there was a dyarthria, which was considered to be dystonic rather than cerebellar or pyramidal in origin. There were involuntary movements in the hands. The right hand had athetoid and myoclonic 
movements. The left was clumsy and apraxic. There was a resistance to passive movements in all four limbs that was thought to resemble gegenhalten. Attempts to sit up in bed induced myoclonic jerks and hypertonic jerks in the legs. Myoclonic jerks were induced in the legs by touch. Plantar responses were flexor. There was no evidence of intellectual impairment.

Investigations included repeated lumbar punctures and two brain MRI scans that were normal. Screening for toxoplasmosis and cryptococcosis was consistently negative. An EEG was diffusely abnormal but did not show any specific features. "Back averaging" was not performed. The myoclonus proved difficult to control and did not respond to a combination of clonazepam, piracetam, carbamazepine, and sodium valproate. The patient became progressively quadriparetic and cachexic. He developed an aspiration pneumonia and died some nine months after initial presentation.

Case 2 was a 38 year old anti-HIV-1 seropositive homosexual man who presented in August 1990 with a four month history of increasing unsteadiness of gait and clumsiness of the right hand. He had been diagnosed anti-HIV-1 seropositive in 1987 after an episode of left sided herpes simplex ophthalmicus. This was complicated by chronic corneal infection and eventually a complete left ptosis was induced with botulinum toxin to protect the cornea. He had pulmonary tuberculosis in 1989. Medications on admission were $250 \mathrm{mg}$ zidovudine twice daily and $300 \mathrm{mg}$ isoniazid, $10 \mathrm{mg}$ pyridoxine, $50 \mathrm{mg}$ dapsone daily and $50 \mathrm{mg}$ fluconazole daily.

General physical examination was normal. On neurological examination he was alert and orientated. Speech was normal. He had a wide based ataxic gait. Cranial nerve examination showed a left ptosis and a scarred left cornea. There was absence of light perception in the left eye and the left fundus could not be visualised. There was $6 / 6$ visual acuity in the right eye with a full visual field, normal pupillary response, and a normal fundus. There was first degree nystagmus looking to the right. Also, there was dysmetria on finger-nose and heel-shin testing in all four limbs; this was more pronounced on the right than on the left. There was dysdiadochokinesis in the right hand. The remainder of the examination was normal.

Screening tests were negative for toxoplasmosis and cryptococcosis and analysis of CSF only showed a raised protein content. CT of the brain was normal and MRI showed diffuse atrophy but no focal lesion above or below the tentorium.

The cerebellar syndrome progressed to the point of making the patient confined to bed. $\mathrm{He}$ did not develop any other neurological disorder and died three months after the initial admission.

\section{PATHOLOGICAL METHODS}

After macroscopic examination blocks from many regions of the brains, including cerebral hemispheres, cerebellar hemispheres, basal ganglia and brainstem were processed for celloidin (case 1) or paraffin (cases 1 and 2) embedding. Sections were stained with routine methods.

Immunohistochemistry was performed on paraffin sections by an indirect immunoalkaline phosphatase (APAAP) method with polyclonal antibodies raised against glial fibrillary acidic protein (GFAP) (Dakopatts, France), Toxoplasma gondii (ICN) and herpes simplex virus (HSV) type 1 and 2 (Dakopatts), and monoclonal antibodies raised against cytomegalovirus (CMV) (Biosoft, France) and varicella zoster virus (VZV) (Biosoft). HIV-Antigens were sought with monoclonal antibodies against p24 and p17 (DuPont, UK).

In situ hybridisation was performed on paraffin embedded material after enzymatic proteolysis using buffered proteinase $K$ at $37^{\circ} \mathrm{C}$ with a biotylinated probe from the entire genome of JC virus cloned onto the site Eco R1 of pBR322 (Enzo Diagnostic, France) at a dilution of 1/5. DNA was denatured at $95^{\circ} \mathrm{C}$ for 30 minutes. The slides were subsequently hybridised overnight at $42^{\circ} \mathrm{C}$. Biotin was visualised with alkaline streptavidine-phosphatase stained by nitro blue tetrazolium/5-bromo-4-chloro-3-indolylphosphate.

\section{PATHOLOGY}

In case 1 macroscopic examination showed old, necrotic changes with an orange yellow discolouration involving the entire cortex in the severely affected areas and limited to the $\mathrm{U}$ fibres in less severely affected regions. These mainly involved the precentral and postcentral gyri and to a lesser extent the superior temporal gyri. Similar changes also involved the claustrum bilaterally. The underlying white matter, basal ganglia, brainstem, and cerebellum were macroscopically unaffected. This appearance was emphasised in a hemispheric section embedded in colloidon and stained with the Loyez method for myelin. This showed a "moth-eaten" appearance of subcortical and cortical myelin with sparing of myelin in the gyri and centrum semiovale (fig 1).

At light microscopy the changes were more extensive than they appeared on gross examination and included demyelination, scanty perivascular mononuclear inflammatory infiltrates, reactive astrocytosis with large bizarre astrocytes, and numerous enlarged oligodendrocytes with inclusions. In the macroscopically affected areas, the lesions had a chronic cystic appearance with rare inclusions bearing oligodendrocytes at the periphery of necrotic areas. More recent changes consisted of focal demyelination with pronounced inflammation and numerous inclusions bearing oligodendrocytes present throughout the $U$ fibres, from the frontal lobes to the calcarine sulci.

By contrast the underlying white matter was normal and devoid of changes due to progressive multifocal leukoencephalopathy, except for secondary degenerative changes 
Figure 1 Case 1:

Coronal section of right cerebral hemisphere at the level of the thalamus showing "moth-eaten" appearance of the subcortical and cortical myelin (arrowed) (Loyez stain).

Figure 2 Case 2: Photomicrograph of cerebellar hemisphere showing severely reduced granular cell layer and virtual disappearance of myelin. (Luxol fast blue/Nissl stain; originally $\times 48)$.

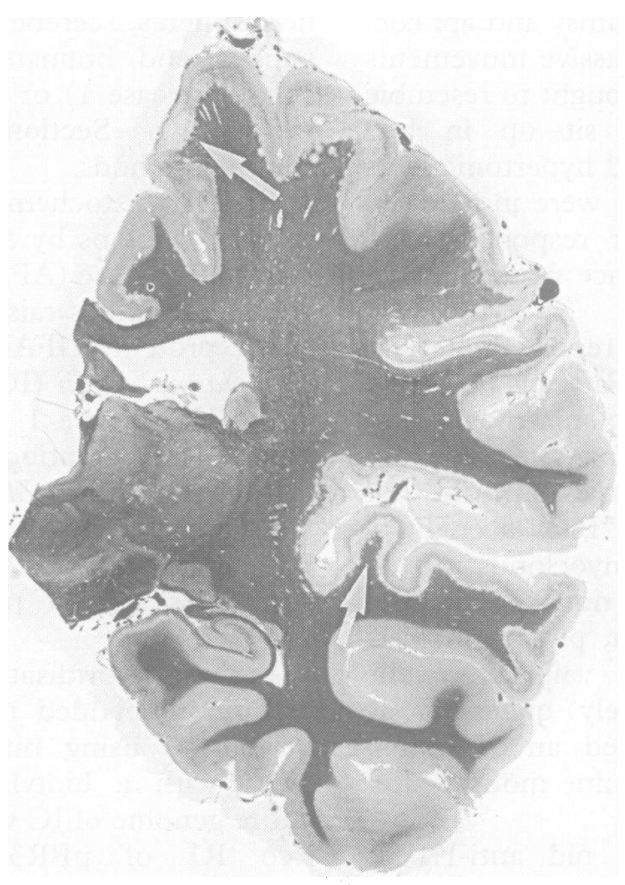

related to the more chronic necrotic cortical areas.

Moderate numbers of Alzheimer type II astrocytes were present in the basal ganglia but no changes due to progressive multifocal leukoencephalopathy were seen in the striatum, globus pallidus, thalamus, nucleus subthalamicus, red nucleus, and substantia nigra of both sides. The brainstem and cerebellum were unremarkable.

In case 2 there was no macroscopic abnormality visible on the hemispheres. Coronal sectioning showed normal ventricles, cortical ribbon, and deep grey nuclei. The cerebellum seemed slightly shrunken and the folia were necrotic in places.

On histological examination the only abnormalities were in the gyri of the cerebellum. There was a diffuse abnormality in the

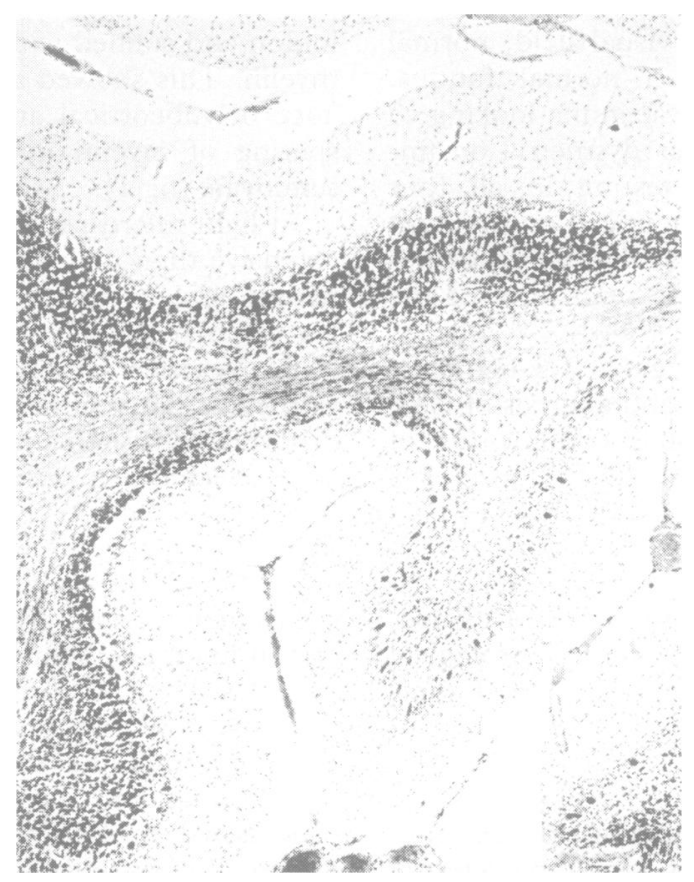

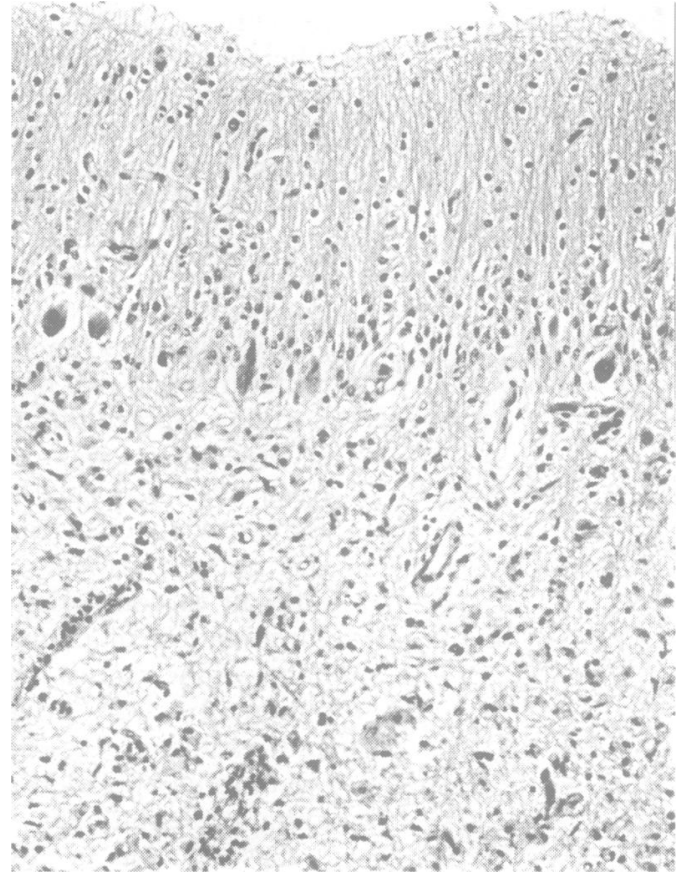

Figure 3 Case 2: Detail of cerebellar cortex showing patchy disappearance of Purkinje cells, hyperplasia of Bergman glia, gliosis of the molecular layer, and complete disappearance of granular cells. (haemotoxylin-eosin stain, originally $\times 120)$.

cerebellar cortex with thinning or loss of large areas of the granular cell layer (fig 2). There were reduced numbers of Purkinje cells and hypertrophy of the Bergman glia was conspicuous (fig 3). The adjacent molecular layer had increased cell density, predominantly composed of astrocytes. The white matter in abnormal folia showed vacuolation and variable amounts of myelin loss (fig 2). There were many macrophages around the smaller blood vessels. Astrocyte numbers were again increased and many were pleomorphic. Enlarged, round nuclei with amphophilic inclusion bodies were visible throughout the abnormal areas including grey matter. A few perivascular inflammatory cells were present. The deep cerebellar white matter, including the dentate nucleus, was spared.

In both cases in situ hybridisation with a probe for papovavirus showed numerous inclusions throughout the affected white

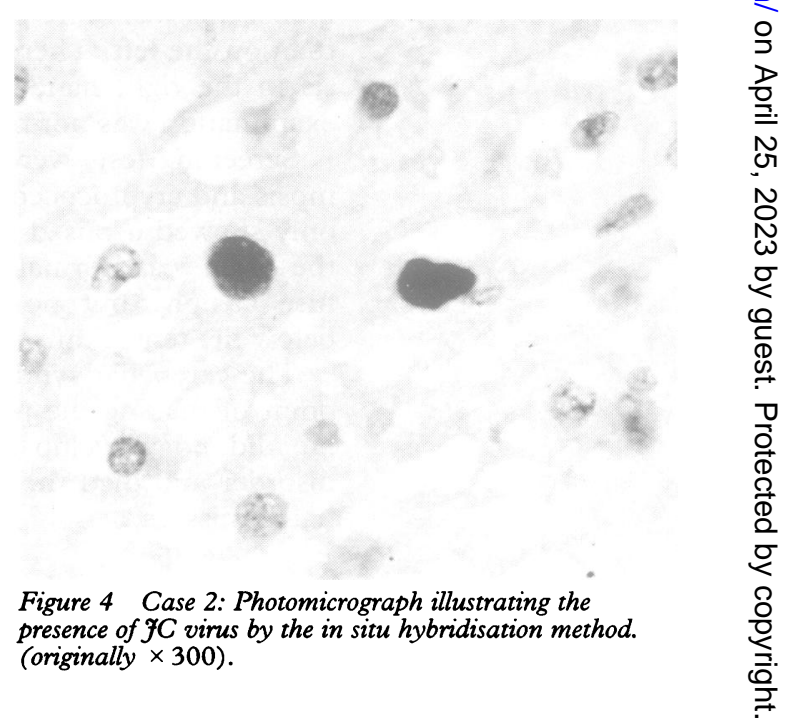


matter and also through all the layers of overlying grey matter (fig 4). Staining for p24 antigen was negative. There were no other focal lesions, micro-organisms were not detected on sections stained or immunostained for viruses, bacteria, fungi, or parasites. There were no multinucleated giant cells and immunostaining for HIV was negative.

\section{Discussion}

These cases are the first to record an association between a progressive neurological disorder and predominant involvement of the grey matter and immediately subcortical structures with JC virus infection without evidence of white matter disease on MRI. Case 1 presented with involuntary movements-namely, myoclonus and athetosis, and then developed progressive quadriparesis. Case 2 had a progressive cerebellar syndrome.

There have been previous reports on pathological and clinical evidence suggesting that progressive multifocal leukoencephalopathy can cause abnormalities in grey matter. Richardson in 1961 and more recently Kuchelmeister and colleagues have described abnormal cells in the granular cell layer in the cerebellum of patients with adjacent white matter progressive multifocal leukoencephalopathy with and without AIDS. ${ }^{1011}$ These cells had nuclei twice the size of normal granular cell nuclei and had little cytoplasm. Kuchelmeister $e t a l^{11}$ did not find any local granular cell layer infection with JC virus in these cases. These authors did not find a reduced number of granular cells. We did not see these cells in our cases.

Clinical reports of patients with progressive multifocal leukoencephalopathy developing seizures and extrapyramidal disorders have considered grey matter involvement although without gross grey matter disease at postmortem. ${ }^{6}$ Hansman Whiteman et al have recently reported an abnormal signal from deep cerebral grey matter on MRI of patients with AIDS and progressive multifocal leukoencephalopathy. ${ }^{12}$ Pathologically these changes were related to progressive multifocal leukoencephalopathy in adjacent white matter and to destruction of myelinated fibres passing through the basal ganglia structures. As we have already indicated MRI was negative in our patients.

In a histological study Aksamit et al found JC virus by in situ hybridisation in satellite oligodendrocytes in grey matter overlying white matter foci in four of 10 patients with AIDS associated progressive multifocal leukoencephalopathy and in glial cells in the granular cell layer of the cerebellum in two patients with progressive multifocal leukoencephalopathy not associated with AIDS.?
These findings were not associated with obvious histopathological abnormality as in the cases described here.

To the clinician these cases represent a hitherto unrecognised presentation of JC virus infection. The classic features of white matter involvement without mass effect or contrast enhancement on CT or MRI were absent and thus there was no evidence from previous knowledge to suggest progressive multifocal leukoencephalopathy as a diagnosis. ${ }^{2}$ This may question the use of the term "leukoencephalopathy" which implies white matter involvement as an essential feature of this disease. Perhaps adoption of the term "JC virus encephalitis" would prevent this misunderstanding in the future.

In conclusion, we highlight the unusual features of these cases of progressive multifocal leukoencephalopathy: predominant infection of the cortex and adjacent white matter and lack of imaging abnormality. Both of the patients had AIDS. This pattern of clinical and pathological involvement has to our knowledge not previously been reported in progressive multifocal leukoencephalopathy not associated with HIV-1.

BJS and HM are funded by the Medical Research Council of Great Britain. We would like to thank Dr B De Toffol for access to the clinical description of case 1 .

1 Åström KE, Mancall EL, Richardson Jr EP. Progressive multifocal leukoencephalopathy. A hitherto unrecognised complication of chronic lymphatic leukaemia and Hodgkin's disease. Brain 1958;81:93-111.

2 Sweeney BJ, Miller RF, Harrison MJG. Progressive multifocal leukoencephalopathy. $B r \mathcal{F}$ Hosp Med 1993;50 187-92.

3 Scaravilli F, Gray F, Mikol J, Sinclair E. Progressive multifocal leukoencephalopathy. In: Scaravilli F, ed. The neuropathology of HIV infection. Berlin: Springer Verlag, 1993:113-7.

4 Holman RC, Janssen R, Buehler JW, Zelasky BS, Hooper WC. Epidemiology of progressive multifocal leukoencephalopathy in the United States: analysis of national mortality and AIDS surveillance data. Neurology 1991;41:1733-6.

5 Miller JR, Barrett RE, Britton CB, et al. Progressive multifocal leukoencephalopathy in a male homosexual with T-cell immune deficiency. $N$ Engl $f$ Med 1982;307: 1436-8.

6 Berger JR, Kaskovitz B, Donovan Post MJ, Dickinson G Progressive multifocal leukoencephalopathy associated with human immunodeficiency virus infection. Ann Intern Med 1987;107:78-87.

7 Aksamit AJ, Gendelman HE, Orenstein JM, Pezeshkpou RGM. AIDS-associated progressive multifocal leukoencephalopathy (PML): Comparison to non-AIDS PML with in situ hybridisation and immunohistochemistry. Neurology 1990;40:1073-78.

8 Vazeux $R$, Cumont M, Girard PM, et al. Severe encephalitis resulting from co-infections with HIV and JC virus. Neurology 1990;40:944 48 .

9 Tada H, Rappaport J, Lashgari M, Amini S, Wong-Stall $F$, Khalili K. Trans-activation of the JC virus late promoter by the TAT protein of type 1 human promoter by the TAT protein of type 1 human immunodeficiency virus in

10 Richardson EP Jr. Progressive multifocal leukoencephalopathy. N Engl f Med 1961;265:815-823.

11 Kuchelmeister K, Bergmann M, Gullotta F. Cellula changes in the cerebellar granular layer in AIDS-associated PML. Neuropathol Appl Neurobiol 1993;19. 398-401.

12 Hansman Whiteman ML, Donovan Post MJ, Berger JR Tate LG, Bell MD, Limonte LP. Progressive multifoca leukoencephalopathy in $47 \mathrm{HIV}$-seropositive patients: neuroimaging with clinical and pathological correlation. Radiology 1993;187:233-40. 